\title{
Feasibility and clinical utility of ambulatory cough monitoring in an outpatient clinical setting: a real-world retrospective evaluation
}

\author{
Anne E. Vertigan ${ }^{1,2}$, Sarah L. Kapela $\mathbb{D}^{1}$, Surinder S. Birring ${ }^{3,4}$ and Peter G. Gibson ${ }^{2,5,6}$
}

${ }^{1}$ Speech Pathology, John Hunter Hospital, New Lambton Heights, NSW, Australia. ${ }^{2}$ Priority Centre for Healthy Lungs, The University of Newcastle Hunter Medical Research Institute, New Lambton, NSW, Australia. ${ }^{3}$ Respiratory Medicine, King's College Hospital, London, UK. ${ }^{4}$ Dept of Respiratory Sciences, King's College London, London, UK. ${ }^{5}$ Centre of Excellence in Severe Asthma, The University of Newcastle Faculty of Health and Medicine, Callaghan, NSW, Australia. ${ }^{6}$ Dept of Respiratory and Sleep Medicine, John Hunter Hospital, New Lambton Heights, NSW, Australia.

Corresponding author: Anne E. Vertigan (anne.vertigan@health.nsw.gov.au)

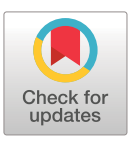

Copyright @The authors 2021

This version is distributed under the terms of the Creative Commons Attribution Non-Commercial Licence 4.0. For commercial reproduction rights and permissions contact permissions@ersnet.org

This article has supplementary material available from openres.ersjournals.com

Editorial comment in ERJ Open Res 2021; 7: 00545-2021 [https://doi.org/10.1183/ 23120541.00545-2021]

Received: 7 May 2021 Accepted: 9 July 2021

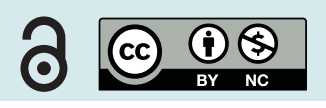

Shareable abstract (@ERSpublications)

Ambulatory cough monitoring provides useful clinical information, including cough frequency and patterns of cough. Logistical issues may preclude use but it can enable measurement of change following treatment and comparison with patient symptoms. https://bit.ly/3Aam4xt

Cite this article as: Vertigan AE, Kapela SL, Birring SS, et al. Feasibility and clinical utility of ambulatory cough monitoring in an outpatient clinical setting: a real-world retrospective evaluation. ERJ Open Res 2021; 7: 00319-2021 [DOI: 10.1183/23120541.00319-2021].

\section{Abstract}

Research question Objective quantification of cough is rarely utilised outside of research settings and the role of cough frequency monitoring in clinical practice has not been established. This study examined the clinical utility of cough frequency monitoring in an outpatient clinical setting.

Methods The study involved a retrospective review of cough monitor data. Participants included 174 patients referred for treatment of cough and upper airway symptoms (103 chronic cough; 50 inducible laryngeal obstruction; 21 severe asthma) and 15 controls. Measures, taken prior to treatment, included 24-h ambulatory cough frequency using the Leicester Cough Monitor, the Leicester Cough Questionnaire and Laryngeal Hypersensitivity Questionnaire. Post-treatment data were available for 50 participants. Feasibility and clinical utility were also reported.

Results Analysis time per recording was up to $10 \mathrm{~min}$. $75 \%$ of participants could use the monitors correctly, and most (93\%) recordings were interpretable. The geometric mean cough frequency in patients was $10.1 \pm 2.9$ (mean $\pm \mathrm{SD}$ ) compared to $2.4 \pm 2.0$ for healthy controls $(\mathrm{p}=0.003$ ). There was no significant difference in cough frequency between clinical groups $(\mathrm{p}=0.080)$. Cough frequency decreased significantly following treatment $(\mathrm{p}<0.001)$. There was a moderate correlation between cough frequency and both cough quality of life and laryngeal hypersensitivity. Cough frequency monitoring was responsive to therapy and able to discriminate differences in cough frequency between diseases.

Conclusion While ambulatory cough frequency monitoring remains a research tool, it provides useful clinical data that can assist in patient management. Logistical issues may preclude use in some clinical settings, and additional time needs to be allocated to the process.

\section{Introduction}

Chronic cough is a commonly occurring problem [1] that impacts quality of life and health resource utilisation [2]. Cough frequency monitoring is one of several tools available to assess cough. While it provides objective quantification of cough frequency, it is rarely utilised outside of research settings, and therefore the clinical utility of cough frequency monitoring in clinical practice has not been established [3].

Ambulatory cough frequency monitoring is more valid and reliable than patient recall and clinician estimation [4, 5]. Acoustic cough measurement tools are designed to discriminate cough sounds from environmental noise [6]. Some tools have also attempted to measure force, intensity and acoustic properties 
of cough; however, cough frequency is the most commonly used [3]. Cough frequency monitoring distinguishes patients with chronic cough from healthy controls [7], measures change following treatment and has been used to demonstrate efficacy of treatment in clinical trials [8,9]. Cough monitoring devices provide valid assessments of cough frequency and correlate well with human counts of cough.

Currently, the most commonly used systems for ambulatory cough frequency monitoring are the Leicester Cough Monitor [4] and the VitaloJAK [10, 11]. Both systems have large data files for later analysis [3]. The Leicester Cough Monitor is a digital ambulatory cough monitor that records sound continuously from an external free-field microphone onto a digital sound recorder at a sampling frequency of $16 \mathrm{kHz}$ and with an encoding bit rate of $64 \mathrm{kbit} \cdot \mathrm{s}^{-1}$ [4]. The Leicester Cough Monitor has good sensitivity (91\%) and specificity (99\%) for detecting cough while filtering background noise and provides repeatable and valid measures. The VitaloJAK was designed specifically for recording cough sounds. It is portable, uses an external microphone, chest sensor and battery pack, and information is stored on a Secure Digital (SD) card. These devices provide 24-h recording which enables assessment during the patient's routine activities. Both systems have been used in previous studies of chronic cough, COPD and acute cough following upper respiratory tract infection [12-14]. The Leicester Cough Monitor has near-automated software and is reported to require a mean of $5 \mathrm{~min}$ operating time per analysis. The VitaloJAK requires a manual assessment of condensed recordings and has a mean operating time of 87 min per analysis [7]. The two systems have different strengths and weaknesses but lead to similar cough counts [3].

We aimed to investigate the feasibility of cough monitoring in a real-world setting by assessing: 1) patient set-up time, completion of recordings and analysis time; 2) cough frequency in a range of respiratory conditions in comparison with healthy controls; and 3) ability to detect a response following speech pathology intervention.

\section{Methods}

This study was conducted in an outpatient clinic at a tertiary referral hospital. It involved a cross-sectional retrospective review of cough monitor data collected as part of previous research studies and contained in a cough monitor database. Patients were referrals to speech pathology between 2010 and 2018 for behavioural management of upper airway symptoms that persisted despite previous medical treatment. These studies were approved by the Hunter New England Human Research Ethics Committee (11/11/16/ 3.04; 14/09/10/4.03; 09/08/19/5.02), and written consent was obtained from all research participants.

\section{Participants}

Participants included 174 patients with cough and upper airway symptoms referred for speech pathology management and 15 healthy controls. Patients included 103 with chronic refractory cough, 50 with inducible laryngeal obstruction and 21 with severe asthma (table 1). Participants with chronic cough had cough persisting despite guideline-based treatment for chronic cough and no lung disease. Inducible laryngeal obstruction was defined as intermittent laryngeal obstruction confirmed by laryngoscopy resulting in symptoms of dyspnoea. Severe asthma was defined as persisting symptoms despite high-dose treatment with inhaled corticosteroids and long-acting $\beta$-agonist. Exclusion criteria included current smoking, untreated asthma, rhinosinusitis, or gastroesophageal reflux disease and lung pathology. Healthy controls were non-smoking with no history of cough, respiratory or voice disorders.

\section{Measures}

24-h ambulatory cough frequency monitoring was conducted using the Leicester Cough Monitor (figure 1). This monitor was chosen as it was the system available in the clinic. The monitor was attached and worn for a 24-h period prior to speech pathology intervention. Participants were encouraged to continue their regular activities for the duration of the recordings. Participants returned the cough monitors at their next appointment visit where the recordings were downloaded, stored and analysed. Three different speech pathologists analysed the cough recordings according to standardised protocols. Cough monitoring was repeated at the conclusion of speech pathology intervention, where possible, and post-treatment data are available for 50 participants. Speech pathology intervention consisted of between one and four sessions with a qualified speech pathologist. It included education, exercises to suppress cough, vocal hygiene training and psychoeducational counselling as previously described [15]. The interval between pre- and post-treatment recordings was between 3 and 4 months.

Cough monitor data were analysed to determine: 1) the number of pre-treatment coughs/hour; 2) the number of post-treatment cough events per hour; 3) total number of daytime cough events; 4) number of daytime coughs per hour; 5) total number of overnight coughs; and 6) number of overnight coughs per hour. Night-time cough was defined from 00:00 to 05:59 h and daytime cough defined from 06:00 to 11:59 h. 


\begin{tabular}{|c|c|c|c|c|}
\hline & $\begin{array}{l}\text { Healthy } \\
\text { controls }\end{array}$ & $\begin{array}{l}\text { Chronic } \\
\text { cough }\end{array}$ & $\begin{array}{c}\text { Inducible laryngeal } \\
\text { obstruction }\end{array}$ & $\begin{array}{l}\text { Severe } \\
\text { asthma }\end{array}$ \\
\hline Subjects & 15 & 103 & 50 & 21 \\
\hline Age years & $51.69 \pm 12.71$ & $60.56 \pm 13.95$ & $58.3 \pm 12.33$ & $52.25 \pm 3.86$ \\
\hline Sex (\% female) & 69 & 70 & 76 & 75 \\
\hline $\mathrm{FEV}_{1} \%$ predicted & $105.69 \pm 16.22$ & $86.4 \pm 15.68$ & $81.21 \pm 16.70$ & $67.63 \pm 18.17$ \\
\hline FVC \% predicted & $106.85 \pm 15.44$ & $85.23 \pm 17.08$ & $85.79 \pm 23.87$ & $85.84 \pm 19.29$ \\
\hline FER \% & $101.08 \pm 10.47$ & $100.07 \pm 12.27$ & $94.14 \pm 14.55$ & $72.17 \pm 16.27$ \\
\hline Duration of cough months & 0 & $109 \pm 125$ & $83 \pm 160$ & $94 \pm 202$ \\
\hline \multicolumn{5}{|l|}{ Comorbid diagnoses } \\
\hline Gastroesophageal reflux & 0 & 57 & 28 & 10 \\
\hline Asthma & 0 & 16 & 15 & 20 \\
\hline Rhinosinusitis & 0 & 41 & 16 & 15 \\
\hline Obstructive sleep apnoea & 0 & 12 & 8 & 2 \\
\hline \multicolumn{5}{|l|}{ Smoking status } \\
\hline Never-smoker & 13 & 63 & 35 & 10 \\
\hline Ex-smoker & 0 & 33 & 11 & 9 \\
\hline Current smoker & 0 & 1 & 0 & 1 \\
\hline Asthma Control Questionnaire & NA & NA & NA & $2.4 \pm 1.2$ \\
\hline Beclomethasone equivalent dose & NA & NA & NA & $1375 \pm 614$ \\
\hline
\end{tabular}

Patient-reported symptom scales included the Leicester Cough Questionnaire [16] and Newcastle Laryngeal Hypersensitivity Questionnaire [17].

Data analysis

Cough frequency data were analysed to compare patients and healthy controls and to investigate differences between clinical groups. Cough monitor results were also compared with Leicester Cough Questionnaire and Laryngeal Hypersensitivity Questionnaire scores to determine the correlation between
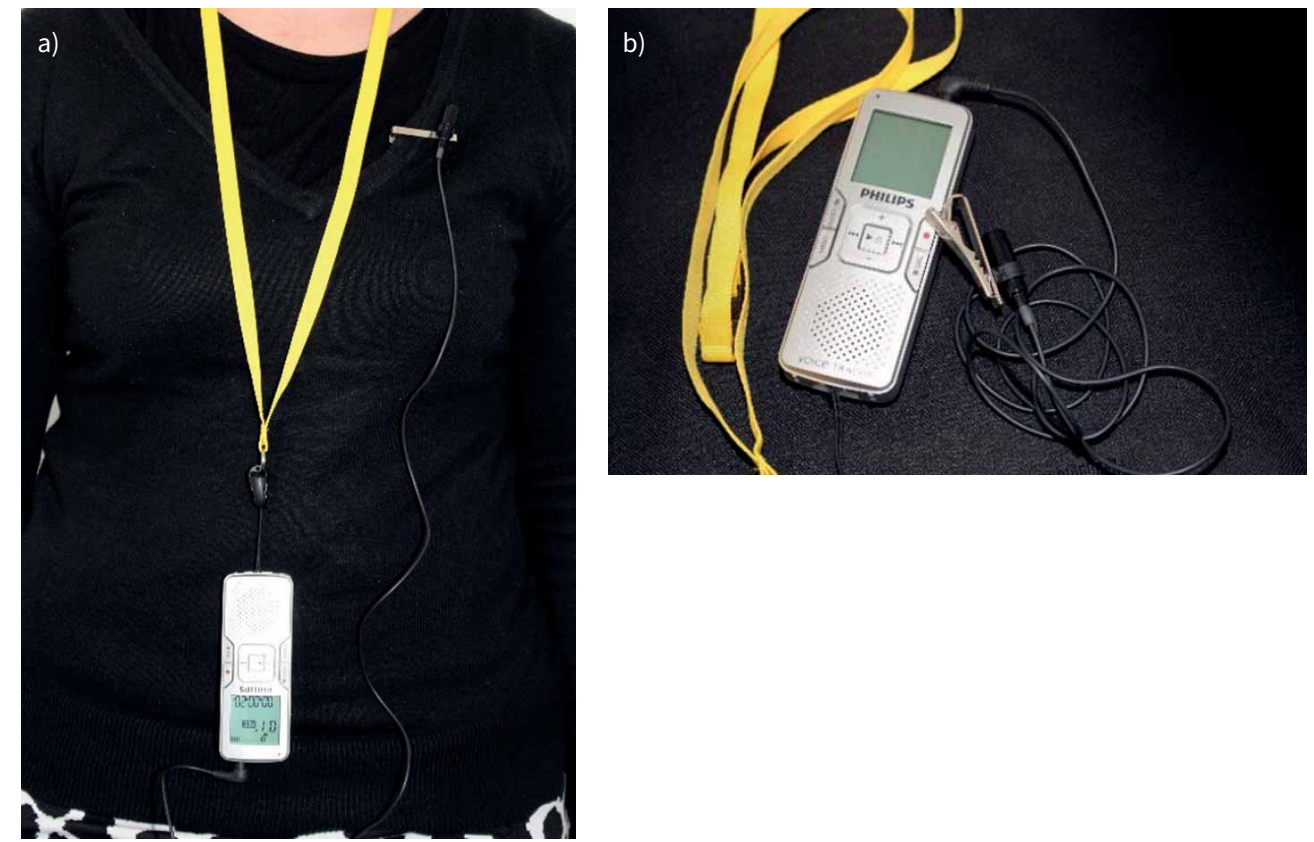

FIGURE 1 a) Photo of the Leicester Cough Monitor as worn by an individual. b) Photo of the Leicester Cough Monitor. 
cough frequency and laryngeal hypersensitivity and between cough frequency and cough quality of life. Data were analysed using mean and standard deviation, geometric mean (GEM) and geometric standard deviation (GSD) or median and interquartile range as appropriate. GEM and GSD were chosen for cough frequency data as the distribution was skewed and required log transformation. Group comparisons were made using t-tests and one way ANOVA as appropriate. Correlations were conducted using a Pearson correlation. Pre-post data were analysed with a paired t-test. Significance was accepted as $\mathrm{p}<0.05$.

\section{Results}

\section{Feasibility}

Equipment and recordings

Most (n=176; 93\%) pre-treatment recordings were analysable, while 13 (7\%) pre-treatment recordings were uninterpretable; two healthy controls and 11 patients. This left 97 recordings available for analysis for the participants with chronic cough, 46 for ILO, 20 for severe asthma and 13 for healthy controls. There was only one uninterpretable post-treatment recording. Over the course of 6 years, 12 monitors were not returned by participants and eight ceased to function. The median life span of monitors was $2-3$ years. 49 (28\%) of the recordings were not contained in a single 24-h report due to patients turning monitors off accidently or when the voice tracer device turned off for unknown reasons.

\section{Set-up time}

The tasks, estimated time and frequency for each patient are summarised in table 2. Set-up tasks included checking batteries, printing instructions, testing and explaining the instructions to the patients. Additional time (up to $1 \mathrm{~h}$ ) was required for troubleshooting/phone calls from nine (6\%) participants.

Analysis

Time required to download, save, rename and analyse each sound file took 5-10 min of clinician time in most cases. However, it took up to $20 \mathrm{~min}$ in 23 cases where there were interruptions to recordings. Additional time was required for software analysis which occurred in the background and did not require direct clinician time.

\section{Acceptability to patient}

Participants were open to wearing a cough monitor, and an estimated $75 \%$ could understand the instructions and use the monitors easily.

\section{Acceptability to clinician}

Formal measures of clinician acceptability were not taken; however, treating clinicians found the clinical inclusion of cough frequency data in patient reports to be of benefit. Specific times and patterns of cough frequency facilitated treatment planning and enabled cough suppression strategies and exercises to be implemented during at-risk periods. Anecdotally, there was less fail to attend for follow-up appointments if provided with a cough monitor - possibly as they had to return it. Cough frequency data were helpful in monitoring therapy progress and feeding back to the clinician.

\section{Utility}

Cough frequency measures available for each individual patient are summarised in table 3. Cough frequency can be summarised graphically to indicate the time distribution of cough events (figure 2). These measures can be taken at baseline and post-treatment to measure change. Objective cough counts can be compared to daily activities such as getting out of bed, to an individual's perception of their cough

\section{TABLE 2 Time required for cough monitoring analysis}

\begin{tabular}{lll} 
Task & Estimated time & Frequency \\
\hline Set-up equipment & $5-10 \mathrm{~min}$ & Per patient visit \\
Explaining use of cough monitor for patient & $5-10 \mathrm{~min}$ & Per patient visit \\
Save/download/rename & $5 \mathrm{~min}$ & Per patient visit \\
Analysis with input from clinician & $5-20 \mathrm{~min}$ & Per patient visit \\
Software processing & $\mathrm{Up} \mathrm{to} 55 \mathrm{~min}$ & Per patient visit \\
Maintaining records of cough monitor allocation & $2 \mathrm{~min}$ & Per patient visit \\
Processing and cleaning returned monitors & $5 \mathrm{~min}$ & Per patient visit \\
Chasing up lost/broken monitors & Variable & Variable \\
Problem solving for difficult to analyse recordings & Variable & Variable \\
\hline
\end{tabular}


TABLE 3 Data available from ambulatory cough monitoring for an individual patient

\begin{tabular}{ll} 
Measure & Example/definition \\
\hline Cough count & The total number of coughs in a designated period of time \\
Cough frequency & The number of coughs per unit of time (usually hours) \\
Cough patterns & Changes in cough frequency per hour \\
\hline
\end{tabular}

frequency and to validated questionnaires. Cough frequency can be measured for a full 24-h period or for select periods of time such as awake versus asleep. The graph generated by cough detection software can show whether cough events occur constantly throughout the day or whether there is a fluctuating pattern.

\section{Cough frequency}

Cough frequency was compared between clinical groups. The 24-h cough frequency, number of daytime and night-time coughs, and daytime and night-time coughs per hour are summarised in table 4.

\section{4-h cough frequency}

The geometric mean (geometric standard deviation) cough events per hour at pre-treatment was 10.1 (2.9) for patients compared to 2.4 (2.0) for healthy controls $(p=0.003)$. There was no significant difference in the cough events per hour between the three clinical groups $(\mathrm{p}=0.080)$.

\section{Daytime cough}

The geometric mean number of daytime cough events for the patient groups was 187.6 (2.5), and there was no significant difference between the clinical groups $(\mathrm{p}=0.187)$. The mean number of daytime coughs per hour for the clinical groups was 10.5 (2.8), and there was no significant difference between groups $(\mathrm{p}=0.088)$.

\section{Overnight cough}

The geometric mean number of overnight coughs for the combined patient groups was 20.9 (4.6), and there was no significant difference between groups $(p=0.794)$. The total number of overnight coughs was significantly lower than the total number of daytime coughs $(\mathrm{p}<0.001)$. The geometric mean overnight coughs per hour was 3.8 (4.5), and there was no significant difference between clinical groups ( $\mathrm{p}=0.531$ ). The number of overnight coughs per hour was significantly lower than the number of daytime coughs per hour $(\mathrm{p}<0.001)$.

\section{Correlation between cough frequency and patient-reported symptoms}

The relationship between cough frequency and other cough outcome measures examined by correlating cough frequency with both laryngeal hypersensitivity and cough quality of life was explored. Scores on the Laryngeal Hypersensitivity Questionnaire range from 3 to 21, and scores below 17.1 are considered abnormal [17]. Lower scores on the Laryngeal Hypersensitivity Questionnaire denote worse laryngeal hypersensitivity. Mean \pm SD baseline Laryngeal Hypersensitivity Questionnaire score was 14.6 \pm SD, which is outside of the normal range. There was a moderately significant negative correlation between cough frequency and laryngeal hypersensitivity $(\mathrm{r}=-0.430, \mathrm{p}<0.001$; figure $3 \mathrm{a})$, suggesting an association between cough frequency and laryngeal hypersensitivity.

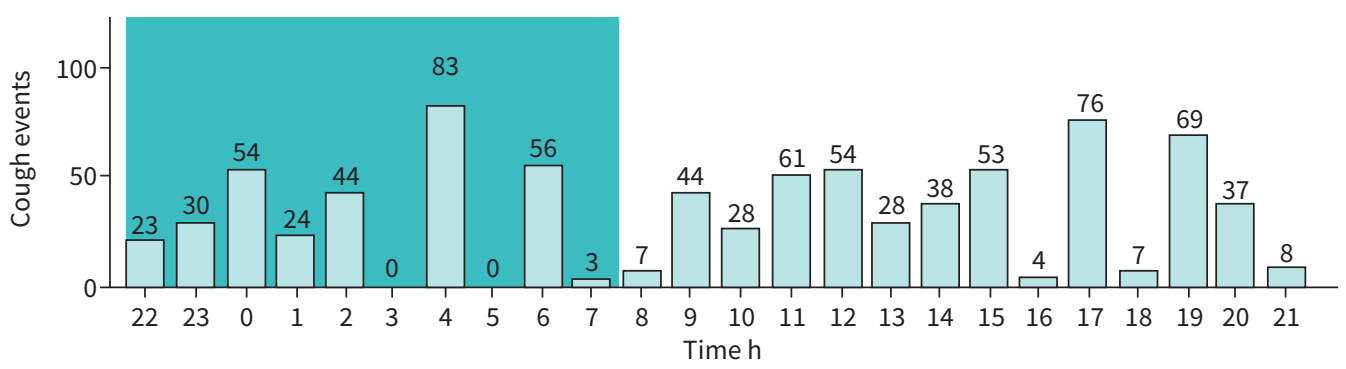

FIGURE 2 An example of time distribution of cough events taken from a single 24-h recording of a participant. 


\begin{tabular}{|c|c|c|c|c|c|}
\hline & $\begin{array}{l}\text { Coughs per } \\
\text { hour }\end{array}$ & $\begin{array}{c}\text { Number of } \\
\text { daytime coughs }\end{array}$ & $\begin{array}{c}\text { Daytime } \\
\text { coughs per h }\end{array}$ & $\begin{array}{c}\text { Number of } \\
\text { overnight coughs }\end{array}$ & $\begin{array}{l}\text { Overnight } \\
\text { coughs per } \mathrm{h}\end{array}$ \\
\hline Chronic cough ( $n=97$ ) & $11.7 \pm 1.1$ & $215.6 \pm 2.5$ & $12.1 \pm 2.8$ & $22.1 \pm 4.7$ & $4.2 \pm 4.6$ \\
\hline ILO (n=46) & $7.5 \pm 2.7$ & $148.6 \pm 2.4$ & $7.9 \pm 2.6$ & $18.3 \pm 4.9$ & $2.9 \pm 4.7$ \\
\hline Asthma $(n=20)$ & $9.5 \pm 2.9$ & $167.0 \pm 2.9$ & $10.7 \pm 2.9$ & $24.4 \pm 3.2$ & $4.6 \pm 2.9$ \\
\hline Healthy controls $(n=13)$ & $2.4 \pm 2.0$ & & & & \\
\hline
\end{tabular}

Scores on the Leicester Cough Questionnaire range from 3 to 21. Lower scores denote worse cough quality of life, and scores below 20 are considered abnormal [16]. Baseline Leicester Cough Questionnaire scores were $12.8 \pm 3.9$, which is also outside the normal range. There was a moderate significant negative correlation between cough frequency and Leicester Cough Questionnaire scores $(\mathrm{r}=-0.430, \mathrm{p}<0.001)$ and cough quality of life $(r=-0.439, \mathrm{p}<0.001$; figure $3 \mathrm{~b}$ ), suggesting an association between cough frequency and cough quality of life.

\section{Ability to detect a response to therapy}

The ability to monitor change after treatment was examined by comparing pre- and post-treatment cough events per hour. The number of cough events per hour reduced significantly between pre- and post-treatment (table 5 and figure S1). This result was consistent with reduction in Leicester Cough Questionnaire and Laryngeal Hypersensitivity Questionnaire scores between pre- and post-assessment (table 5).

\section{Discussion}

This study examined the feasibility and clinical utility of collecting cough monitor data in an outpatient clinical setting and identified the data provided by ambulatory cough frequency monitoring. This is the first paper to discuss clinical utility of ambulatory cough detection monitors in patients with cough and upper airway symptoms referred for speech pathology management. Furthermore, cough frequency monitoring distinguished patients from controls, monitored change following therapy and correlated with
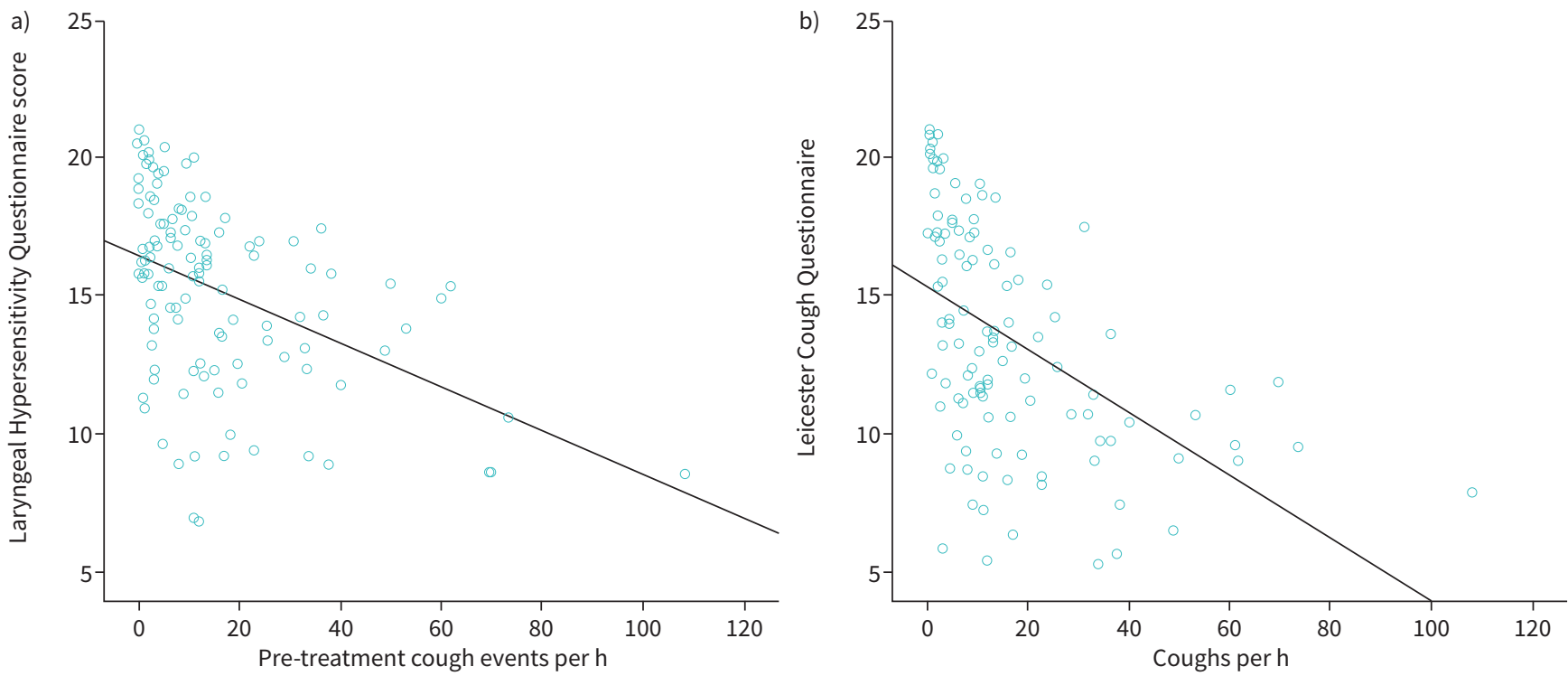

FIGURE 3 a) Scatterplot showing correlation between Laryngeal Hypersensitivity Questionnaire (LHQ) scores and cough frequency. Scores range between 3 and 21, and lower LHQ scores denote worse laryngeal hypersensitivity. b) Scatterplot showing correlation between Leicester Cough Questionnaire (LCQ) scores and cough frequency. Scores range between 3 and 21, and lower LCQ scores denote worse cough quality of life. 


\begin{tabular}{|c|c|c|c|c|c|}
\hline & $\begin{array}{c}\text { Pre } \\
\text { GEM } \pm G S D^{\#}\end{array}$ & $\begin{array}{c}\text { Post } \\
\text { GEM } \pm G S D^{\#}\end{array}$ & $\begin{array}{l}\text { Change } \\
\text { GEM (sE) }\end{array}$ & $95 \% \mathrm{Cl}$ & $\begin{array}{c}\text { p-value }{ }^{q} \text { paired } \\
\text { t-test }\end{array}$ \\
\hline Coughs per $\mathrm{h}$ & $10.5 \pm 3.1^{+}$ & $4.0 \pm 3.4$ & $2.6(1.2)$ & 1.9 to 3.7 & $<0.001$ \\
\hline LCQ & $12.5 \pm 3.3$ & $16.4 \pm 3.5$ & $3.9(0.6)$ & 2.8 to 5.0 & $<0.001$ \\
\hline LHQ & $14.5 \pm 3.1$ & $16.8 \pm 3.2$ & $2.3(0.05)$ & 1.3 to 3.2 & $<0.001$ \\
\hline
\end{tabular}

GEM: geometric mean; GSD: geometric standard deviation; SE: standard error; Cl: confidence interval; LCQ: Leicester Cough Questionnaire; LHQ: Laryngeal Hypersensitivity Questionnaire. ": $n=50$; ๆ: $p$-value was calculated using a paired sample t-test. ${ }^{+}$: the pre-treatment value in table 5 differs from the pre-treatment value reported in table 4 because not all patients had post-treatment cough frequency data.

cough quality of life and laryngeal hypersensitivity. It showed no significant difference in cough frequency between patient groups confirming that cough is increased not only in chronic refractory cough but in other diseases. These results are consistent with the concepts of cough and laryngeal hypersensitivity syndrome. It demonstrates that cough can be present and result from disease but also occur in the absence of identified medical disease $[18,19]$.

It can be clinically useful to compare the objective measure of cough frequency to the person's perception of cough frequency. Ambulatory cough monitoring can bring awareness to the patient especially when cough frequency is underestimated. It can also provide objective feedback to the patient and referring specialist regarding the change in cough as a result of treatment.

A number of logistical issues need to be considered when introducing cough frequency monitoring into clinical practice. Additional time needs to be allocated for tasks associated with the collection and analysis of cough monitoring data (table 2). The cost of the monitor, software and replacement of lost or broken monitors needs to be considered. Costs can be considerable and vary according to the records and setting. A number of monitors may need to be available to enable provision to several patients concurrently. Patients may need to attend additional appointments to return cough monitors following their initial assessment visit or to collect monitors for their post-treatment assessment or returned by post for convenience. In clinical practice patients may be lost to follow-up and may not complete a full course of behavioural intervention, which makes post-treatment measurement difficult. If the cough monitor is provided at the initial assessment visit, the full cough assessment results will not be available immediately, which can delay reporting back to referring clinicians. Another factor to consider is that simply wearing a cough monitor might change behaviour in some patients. For example, patients may avoid wearing the monitors at work or refrain from engaging in certain conversations or activities.

The turnaround time for results could be within 1 or $2 \mathrm{~h}$ of returning the monitor as long as resources were available to download and analyse the recordings. This means that the cough monitor data can be available to assist clinical decision making. Another issue to consider is that cough may be relevant to the patient even if the count is low, and so cough frequency should not be used in isolation from other relevant clinical cough outcome measures. In these situations it is useful to correlate the objective cough count to other cough outcome measures such as cough severity and cough quality of life. In these situations it can be relevant to ask why the cough count is low, for example does the patient only cough in certain situations or is it the severity but not the frequency of cough that concerns the patient, or did the patient choose not to wear the cough monitor in situations that typically provoke cough such as work for confidentiality reasons?

Cough frequency monitoring is commonly performed in research settings where a more comprehensive range of cough measures is employed [7, 20]. It is less likely to occur in clinical practice [7] where the focus is typically on determining the cause of chronic cough.

Cough frequency measures compliment but do not replace other useful measures such as cough quality of life questionnaires and cough severity ratings or cough quality of life [21]. In fact Boulet et al. [21] argue that cough frequency monitoring measures a different construct than other cough measures as there is a poor correlation between cough counts and cough quality of life. While cough frequency may be less relevant to some patients than quality of life measures, it is important in evaluating the outcome of antitussive trials [20]. The correlation between questionnaire scores and cough frequency in the current 
study was not high confirming that patient-reported measures cannot be substituted for objective cough frequency measures.

Limitations of this study include the retrospective design and that it involved analysis of data collected for other purposes. It used convenience sampling. It involved patients enrolled in research studies who may have had better capacity to adhere to operating instructions. Intervention was not part of this study and may not have been standardised. It reflects the outcome in a single clinic, hence the data may be susceptible to bias. Post-treatment cough monitor results were not available for all participants. The cut-off times used to differentiate day and night coughing were arbitrary and may not correspond to actual sleeping times. Further, the duration of daytime cough was longer than overnight cough. Finally, this study only investigated one type of monitor.

Future developments of cough frequency monitoring technology should consider portable, low-cost, real-time operation [3]. Mobile cough detection systems are aiming to differentiate cough from false alarms such as throat clearing, laughter and speech. They should be available over a 24-h period during everyday activity in order to obtain a representative cough frequency with respect to specific disorders and be scalable and cost efficient [22, 23]. Smart phone apps could also prompt patients to rate their cough severity and even include cough quality of life measures. Current cough monitors require dedicated time for data analysis and real-time data are not available. Real-time measurement could reduce time for analysis, reduce the time between data capture and analysis, and provide instant feedback to patients and clinicians. Kvapilova et al. [24] described a system currently in development based on smart phone applications for continuous sound collection and automated detection and analysis of cough sounds. While it would be logistically easier for patients to use their own phones for cough monitoring, KvapiLova et al. warn that applications need further verification and testing. Complex machine learning algorithms can drain battery life [25].

Smart phone cough monitoring technology has been investigated to measure cough quality as a biomarker of asthma severity. Swarnkar et al. [26] analysed 3161 cough episodes in 224 paediatric subjects and showed no/mild disease could be separated from moderate asthma based on cough sound analytic technology. The authors analysed both spontaneous and voluntary coughs and only required five cough samples per patient which could be collected at the time of the patient's hospital visit rather than having to take a cough monitor home with the patient. Overnight coughing has been identified as a potential biomarker for asthma control [27].We argue that exclusive overnight monitoring of cough frequency would not be appropriate for patients with chronic cough due to the low frequency of nocturnal cough.

\section{Conclusion}

Currently available ambulatory cough frequency monitoring tools have capacity to provide useful clinical information for patients with chronic cough including cough frequency and patterns of cough. It enables measurement of change following treatment, comparison with patient-reported symptoms and assists with treatment planning. Logistical issues may preclude routine use in many clinical settings.

Provenance: Submitted article, peer reviewed.

Conflict of interest: A.E. Vertigan has nothing to disclose. S.L. Kapela has nothing to disclose. S.S. Birring reports research funding for cough monitoring paid to King's College Hospital from Gala Therapeutics, ESA Medical and Avalyn Pharma, outside the submitted work. P.G. Gibson has nothing to disclose.

\section{References}

1 Song WJ, Chang YS, Faruqi S, et al. The global epidemiology of chronic cough in adults: a systematic review and meta-analysis. Eur Respir J 2015; 45: 1479-1481.

2 Chamberlain SA, Garrod R, Douiri A, et al. The impact of chronic cough: a cross-sectional European survey. Lung 2015; 193: 401-408.

3 Hall Jl, Lozano M, Estrada-Petrocelli L, et al. The present and future of cough counting tools. J Thorac Dis 2020; 12: 5207-5223.

4 Birring S, Fleming T, Matos S, et al. The Leicester Cough Monitor: preliminary validation of an automated cough detection system in chronic cough. Eur Respir J 2008; 31: 1013-1018.

5 Smith J, Owen E, Earis J, et al. Cough in COPD: correlation of objective monitoring with cough challenge and subjective assessments. Chest 2006; 130: 379-385.

6 Kulnik ST, Williams NM, Kalra L, et al. Cough frequency monitors: can they discriminate patient from environmental coughs? J Thorac Dis 2016; 8: 3152-3159. 
7 Spinou A, Birring SS. An update on measurement and monitoring of cough: what are the important study endpoints? J Thorac Dis 2014; 6: Suppl. 7, S728-S734.

8 Smith JA, Kitt MM, Butera P, et al. Gefapixant in two randomised dose-escalation studies in chronic cough. Eur Respir J 2020; 55: 01615-2019.

9 Smith JA, McGarvey LPA, Badri H, et al. Effects of a novel sodium channel blocker, GSK2339345, in patients with refractory chronic cough. Int J Clin Pharmacol Ther 2017; 55: 712-719.

10 McGuinness K, Holt K, Dockry R, et al. P159 Validation of the VitaloJAK ${ }^{\mathrm{TM}} 24$ hour ambulatory cough monitor. Thorax 2012; 67: Suppl. 2, A131.

11 Mines D, Bacci E, Nguyen AM, et al. Assessment of inter- and intra-rater reliability of objective cough frequency in patients with chronic cough. Eur Respir J 2019; 54: Suppl. 63, PA4342.

12 Deblej Elghamoudi D, Sumner H, McGuiness K, et al. P241 The feasibility and validity of objective cough monitoring in children using an adult cough detection system. Thorax 2015; 70: Suppl. 3, A198.

13 Lee KK, Matos S, Evans DH, et al. A longitudinal assessment of acute cough. Am J Respir Crit Care Med 2013; 187: 991-997.

14 Kanemitsu Y, Smith J, Butera P, et al. The efficacy of bradanicline in refractory chronic cough. Eur Respir J 2020; 56: Suppl. 64, 4564.

15 Vertigan A, Theodoros D, Gibson PG, et al. Efficacy of Speech pathology management for chronic cough: a randomised placebo controlled trial of treatment efficacy. Thorax 2006; 61: 1065-1069.

16 Birring S, Prudon B, Carr A, et al. Development of a symptom specific health status measure for patients with chronic cough: Leicester Cough Questionnaire. Thorax 2003; 58: 339-343.

17 Vertigan AE, Bone SL, Gibson PG. Development and validation of the Newcastle laryngeal hypersensitivity questionnaire. Cough 2014; 10: 1.

18 Song W-J, Chang Y-S, Morice A. Changing the paradigm for cough: does 'cough hypersensitivity' aid our understanding? Asia Pac Allergy 2013; 4: 3-13.

19 Chung K. Chronic 'cough hypersensitivity syndrome': a more precise label for chronic cough. Pulm Pharmacol Ther 2011; 24: 267-271.

20 Birring SS, Spinou A. How best to measure cough clinically. Curr Opin Pharmacol 2015; 22: 37-40.

21 Boulet L-P, Coeytaux RR, McCrory DC, et al. Tools for assessing outcomes in studies of chronic cough: chest guideline and expert panel report. Chest 2014; 145: 794-802.

22 Barata F, Kipfer K, Weber M, et al. (editors). Towards Device-Agnostic Mobile Cough Detection with Convolutional Neural Networks. 2019 IEEE International Conference on Healthcare Informatics (ICHI); 2019, 10-13 June 2019.

23 Porter P, Abeyratne U, Swarnkar V, et al. A prospective multicentre study testing the diagnostic accuracy of an automated cough sound centred analytic system for the identification of common respiratory disorders in children. Respir Res 2019; 20: 81.

24 Kvapilova L, Boza V, Dubec P, et al. Continuous sound collection using smartphones and machine learning to measure cough. Digit Biomark 2019; 3: 166-175.

25 Hoyos-Barcelo C, Monge-Alvarez J, Zeeshan Shakir M, et al. Efficient k-NN implementation for real-time detection of cough events in smartphones. IEEE J Biomed Health Inform 2018; 22: 1662-1671.

26 Swarnkar V, Abeyratne U, Tan J, et al. Stratifying asthma severity in children using cough sound analytic technology. J Asthma 2019; 58: 160-169.

27 Tinschert P, Rassouli F, Barata F, et al. Prevalence of nocturnal cough in asthma and its potential as a marker for asthma control (MAC) in combination with sleep quality: protocol of a smartphone-based, multicentre, longitudinal observational study with two stages. BMJ Open 2019; 9: e026323. 